\title{
Preparing dental students for careers as independent dental professionals: clinical audit and community-based clinical teaching
}

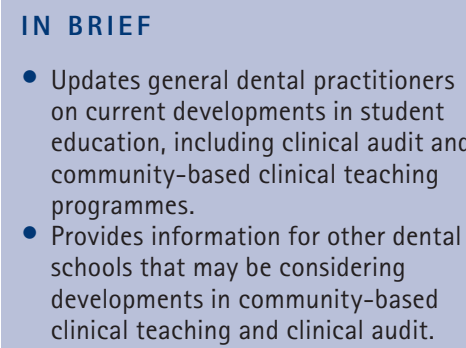

\author{
C. D. Lynch, ${ }^{1}$ J. Llewelyn, ${ }^{2}$ P. J. Ash ${ }^{3}$ and B. L. Chadwick ${ }^{4}$ \\ VERIFIABLE CPD PAPER
}

Community-based clinical teaching programmes are now an established feature of most UK dental school training programmes. Appropriately implemented, they enhance the educational achievements and competences achieved by dental students within the earlier part of their developing careers, while helping students to traverse the often-difficult transition between dental school and vocational/foundation training and independent practice. Dental school programmes have often been criticised for 'lagging behind' developments in general dental practice - an important example being the so-called 'business of dentistry', including clinical audit. As readers will be aware, clinical audit is an essential component of UK dental practice, with the aims of improving the quality of clinical care and optimising patient safety. The aim of this paper is to highlight how training in clinical audit has been successfully embedded in the community-based clinical teaching programme at Cardiff.

\section{INTRODUCTION}

Readers of the $B D J$ and others involved in the provision of dental services will be familiar with the need to implement the principles of clinical governance within their own practice and area of healthcare delivery. ${ }^{1}$ Clinical governance has been defined as 'a framework through which NHS organisations are accountable for continuously improving the quality of their services and safeguarding high standards of care by creating an environment in which excellence in clinical care can flourish'. ${ }^{2}$ Sadly, there are examples of situations and scandals in UK healthcare that occurred before the pre-formalised clinical governance era including unacceptably high post-paediatric cardiac surgery mortality rates and organ retention scandals. It is axiomatic within healthcare

"Senior Lecturer/Honorary Consultant in Restorative Dentistry, Tissue Engineering \& Reparative Dentistry, ${ }^{4}$ Professor/Honorary Consultant in Paediatric Dentistry, School of Dentistry, Cardiff University, Cardiff; ${ }^{2}$ Speciality Dentist, St David's Primary Dental Care Unit, ${ }^{3}$ Consultant/Director of Primary Dental Care Outreach Units, St David's Hospital, Cardiff

${ }^{*}$ Correspondence to: Dr Christopher D. Lynch Email: Iynchcd@cardiff.ac.uk

\section{Refereed Paper}

Accepted 4 March 2011

DOI: 10.1038/sj.bdj.2011.383

${ }^{\circ}$ British Dental Journal 2011; 210: 475-478 that excellence in the quality of care and systems of care must be sought to optimise patient outcomes. One means of ensuring quality of care is via clinical audit, which has been defined as '... the systematic, critical analysis of the quality of dental care, including procedures and processes used for diagnosis, intervention and treatment, the use of resources and the resulting outcome and quality of life as assessed by both professionals and the patient..." ${ }^{3}$ In other words, healthcare practitioners must strive to examine the outcome of their treatments and treatment protocols to ensure that they are 'fit for purpose', meet best standards, and that patients are not exposed to adverse risk, while the outcome of their treatment is optimised.

However, while audit has been formalised in UK dental practice, how well are dental students prepared for this important aspect of their professional careers as dentists? In recent years, dental school education has shifted from being a mere assessment of proficiency or ability at performing prescribed numbers of clinical tasks, to, in addition to the achievement of competence in clinical dentistry, an increased emphasis on the dental student becoming a dental professional. The transition from the dental school environment to the relative shelter of vocational/dental foundation training and subsequently to independent practice has often been considered problematic, ${ }^{4,5}$ with the view often being expressed that dental students 'are no longer as good as they used to be. ${ }^{6}$ Community-based clinical teaching/outreach teaching was introduced in recent years to help this transition from the so-called 'artificial' dental school environment, where students frequently encountered patients whose treatment needs were dissimilar to what they could expect to treat in subsequent general practice, to working in a purpose-built clinic or a series of dispersed general practices, often situated in an area of high need for primary care dentistry. ${ }^{7}$ Community-based clinical teaching has become a success in its own right, with notable UK centres at Kings College London, ${ }^{8}$ Leeds, ${ }^{9}$ Sheffield ${ }^{10}$ and our own at Cardiff. ${ }^{11}$ Data from the Cardiff centre have demonstrated that the teaching programme is popular and has positive impacts on the careers of current and former students, ${ }^{12}$ as well as being associated with an increase in student confidence in performing clinical dentistry. ${ }^{13}$

How does clinical audit 'fit in' to dental school education? Traditionally, features such as clinical audit were seen as part of the 'business of dentistry', and for which 
dental school graduates were poorly prepared. However, recent guidance from regulatory bodies and specialist interest groups involved in dental education have emphasised the need for dental students to be exposed to, and trained in how to, complete clinical audits. The third/ interim edition of the General Dental Council (GDC)'s The first five years states that 'learning opportunities and experiences should enable students to develop an understanding of audit and clinical governance'. ${ }^{14}$ Similarly, the Association for Dental Education in Europe (ADEE)'s Profiles and competences for the European dentist notes that 'on graduation, a dentist must be competent at: audit and clinical governance'. ${ }^{15}$ Clearly, there is a need for UK dental students to be exposed to, and preferably participate in, clinical audit during their dental school training. At Cardiff, this experience is gained during the St David's community-based clinical teaching programme, which takes place during final year. As such, it is believed that working in a close approximation to independent clinical practice in a primary care environment will encourage students to take ownership of the process of audit and come to view that it will have a positive impact on their own clinical practice, rather than simply being a 'chore' to complete. The aim of this paper is to highlight how training in clinical audit has been successfully embedded in the community-based clinical teaching programme at Cardiff.

\section{THE CARDIFF COMMUNITY-BASED CLINICAL TEACHING PROGRAMME}

UK community-based clinical teaching programmes have followed two traditional models:

- The 'dispersed practice' model, as utilised at Sheffield ${ }^{10}$

- The 'purpose-built' model, as utilised at Cardiff, ${ }^{7}$ Kings College London ${ }^{8}$ and Leeds ${ }^{9}$.

Cardiff was one of the early pioneers of the 'purpose-built model' approach, and in 2002, a 12-chair purpose-built unit was opened at the St David's Hospital (Fig. 1). This unit was sited in an area of high-need for primary care dentistry and, unlike the base dental school, is located in a highpopulation area near the city centre. Each

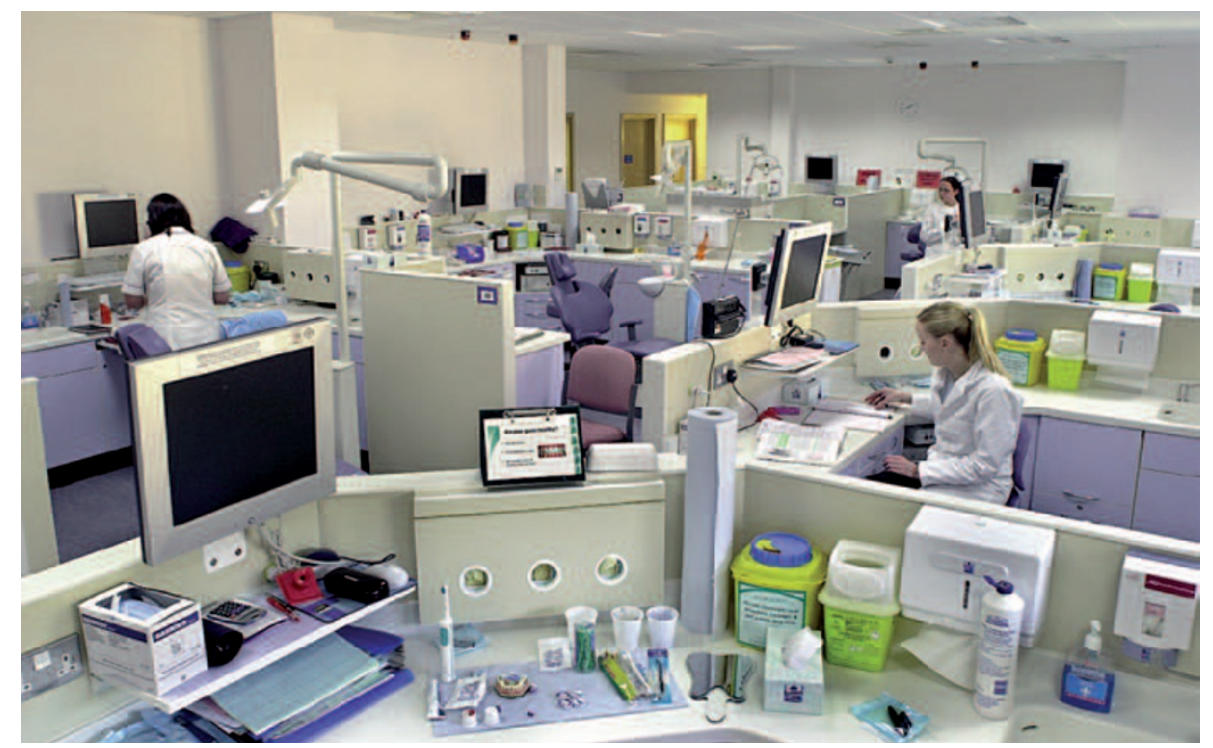

Fig. 1 The dental clinic at the St David's Hospital, Cardiff, where the community-based clinical teaching programme is situated

teaching session typically accommodates ten final year dental students along with two DCP students (dental hygiene and therapy). In contrast to current arrangements at the base dental school, each dental student operator is assisted by a qualified dental nurse. Clinical teaching is delivered by two full-time permanent staff along with visiting teachers from general practice and community settings, as well as some staff from the base dental school. The staff:student teaching ratio is usually $1: 6$. The Cardiff programme is unique as, in contrast to programmes at other schools where outreach schemes are limited to, for example paediatric or restorative dentistry, dental students undertake 'total patient care' across the spectrum of primary care dentistry. Electronic dental records are maintained and clinical treatments are supported by digital radiography. In contrast to the base dental school, dental students are responsible for managing their own appointment book - an important and appropriate responsibility for emerging independent dental practitioners. The positive attributes of the Cardiff/St David's approach include a consistent quality of supervision, consistent nursing support, and consistent access to equipment and dental materials, with the aim of easing transition to subsequent independent practice. Some expansion of the Cardiff programme is imminent - based on the success of the St David's community-based clinical teaching programme, a larger 18 chair unit is currently being built at
Mountain Ash/Cynon Valley - a suburban area, 18 miles away from main population centre in Cardiff city. It is anticipated that this unit will receive its first intake of students in late 2011, doubling the time spent by Cardiff dental students training in the community-based clinical teaching programme.

\section{AUDIT PROJECTS COMPLETED AT THE CARDIFF COMMUNITY-BASED CLINICAL TEACHING PROGRAMME}

Since the opening of the centre in 2002, final year students complete a group audit project over the course of their year at the St David's Primary Dental Care Unit. Students receive didactic teaching in relation to clinical audit within the base dental school towards the end of their fourth year. Within the community-based clinical teaching programme, students are allocated to groups during October of their final year of studies and audit titles are allocated to each group. Working in groups, students collect data over the subsequent months. Groups are supported by a member of the clinical staff who provides advice and helps with important steps such as setting of standards, etc, before the students collect data. The group audit projects are presented to other student colleagues and dental school teaching staff at the base dental school in spring of final year. The projects are judged by a staff panel, including some local vocational/foundation trainers, and a prize awarded to the best project. 
Table 1 Minimum targets in relation to radiographic film quality as identified by the National Radiation Protection Board. (The recommended target should be reached within three years of implementing audit; the 'interim' target should be the minimum achievable standard within a much shorter time)

\begin{tabular}{l|l|l}
\multirow{2}{*}{ Rating } & \multicolumn{2}{|c}{$\%$ of radiographs taken } \\
\cline { 2 - 3 } & Target & $\begin{array}{l}\text { Interim } \\
\text { target }\end{array}$ \\
\hline I & $\geq 70 \%$ & $\geq 50 \%$ \\
\hline II & $\leq 20 \%$ & $\leq 40 \%$ \\
\hline III & $\leq 10 \%$ & $\leq 10 \%$
\end{tabular}

Some examples of recently completed audit projects reported here include:

- Quality assurance of intraoral radiographic techniques

- Crown/bridge impression quality

- Success rate of inferior alveolar nerve block analgesia

- Efficiency of triage procedures at the St David's Primary Dental Care Unit.

\section{Quality assurance of intraoral radiographic techniques}

Optimising quality in undertaking radiographic assessments is important for maintaining patient safety and avoiding unnecessary exposure to harmful ionising radiation. ${ }^{16}$ Let alone this, there are legal requirements for dental practitioners to regularly audit the quality of their radiographic techniques. ${ }^{17}$ Introduction of this particular audit to dental students working in the community-based clinical teaching programme will facilitate their implementation of this audit in their subsequent practices. For this particular audit, 100 intraoral radiographs exposed by student operators within the programme were randomly selected. The quality rating recorded contemporaneously by the operators was noted ( 1 = excellent, 2 = diagnostically acceptable, 3 = unacceptable) and the radiographs were reassessed by the students completing the audit. The standards for the audit were those outlined by the National Radiation Protection Board, which has set minimum targets in relation to radiographic quality (Table 1); these specify 'ideal' and 'interim' targets in relation to the proportion of films assessed which should be in each of the three quality categories. (The recommended target should be reached within three years of implementing audit; the 'interim' target should be the minimum achievable standard within a much shorter time). In this audit, it was noted that there was agreement between the operator and the subsequent examiner in $63 \%$ of cases. Sixty-one percent of films were categorised as Grade 1, $21 \%$ as Grade 2, and $18 \%$ as Grade 3. It was noted that the interim targets were met in relation to films catorgorised as Grade 1 and Grade 2. This is positive given the relative inexperienced state of the operators. As an outcome, and suggested means of improvement of patient care, it was recommended that students receive further training in the use of the radiographic equipment within the unit and that the audit be repeated over the following 12 months.

\section{Crown/bridge impression quality}

Achieving quality in crown and bridge impressions in general dental practice is important, as poor quality impressions can lead to loss of chairside time, loss of patient confidence and increased financial costs in addressing the consequences of recording a poor impression. In addition, poorly fitting crowns and bridges, where cemented, can lead to periodontal disease and/or caries within abutment teeth. Despite the importance of recording accurate and detailed impressions, evidence from general practice indicates that the quality of impressions for crowns and bridges is less than ideal. ${ }^{18-20}$ The aim of this audit was to examine the quality of impressions for crowns and bridges within a dental laboratory. Quality ratings were devised for this audit ( 1 = clinically acceptable with no discrepancies, 2 = some discrepancies but still clinically acceptable, 3 = unacceptable). The standard of 90\% of impressions being satisfactory (quality 1 or 2) was set as the standard for the audit. Fifty impressions were examined (30 maxillary, 20 mandibular). Overall, 23\% of impressions were graded as Quality 1, 56\% as Quality 2 and 21\% as Quality 3. In this regard, the standard for this audit was not met. Recommendations from the audit, with the aim of improving impression quality, included:

- Further training for students of how to make impressions for crowns and bridges

- Better assessment of impressions by supervisors, including the use of a microscope, before dispatching impressions to the laboratory

- Standardisation/training for visiting/ external supervisors to the unit.

It is planned to repeat this audit over the next 12 months.

\section{Success rate of inferior alveolar nerve block analgesia}

Achieving adequate anaesthesia is important for patient comfort during treatment and can avoid unnecessary patient pain and discomfort. However, the delivery of inferior alveolar nerve (IAN) block analgesia can be problematic in dental practice. $^{21,22}$ The aim of this audit was to examine the success rate of IAN block analgesia by student operators within the community-based clinical teaching programme. Based on the available literature, a standard of $85 \%$ success rates was set. $^{21,22}$ The audit was limited to restorative dentistry procedures (eg restorations, crown preparations, etc) and successful anaesthesia was defined as anaesthesia achieved within five minutes of administration and an absence of pain during the procedure. A total of 83 IAN blocks were given, of which 73 (88\%) were successful. Six failures were noted when IAN blocks were delivered to the patient's left, and four failures noted when delivered to the patient's right. Reasons for failure were suspected to relate to anatomical variations and improper positioning technique. While the success rates observed in this audit exceeded the standard set, potential was still identified for further improving success rates. Recommendations made from this audit included a revision of the anatomical structures relevant to the delivery of IAN block analgesia for students before graduation.

\section{Efficiency of triage procedures at the St David's Primary Dental Care Unit}

The St David's Primary Dental Care Unit provides emergency dental care for patients who are registered at the Unit. Patient registration commences when a patient is accepted for treatment and continues beyond completion of treatment to include one recall appointment (determined by the relevant NICE guidelines) at which the patient is viewed to be dentally fit. The service is usually initiated 
by a telephone call from a patient seeking care, which is logged. To enhance the student experience at the community-based clinical teaching programme, emergency patients are allocated an appointment for emergency treatment with a student (usually a cancellation by another patient). Once a suitable time slot is identified, the patient is then called back with appointment details. The aim of this audit was to examine the efficiency of triage procedures used within the emergency care service, on the premise that patients with need for emergency and urgent treatment are seen within the minimum time possible. The standard for the audit adopted was the Scottish Dental Clinical Effectiveness Programme (Emergency Dental Care), ${ }^{23}$ which suggests minimum times for providing patients with emergency care based on their clinical need. These include:

- Emergency Care (eg uncontrollable dental haemorrhage following dental extraction, swelling around throat or eyes, trauma confined to the dental arches) - seen within one hour of presentation or calling the unit

- Urgent Care (severe dental and facial pain not controlled by over-the-counter medications, dental and soft tissue acute infections, fractured teeth/teeth with pulpal exposures) - seen within 24 hours of presentation or calling the unit

- Routine Care (mild/moderate dental pain that responds to over-the-counter medications, debonded restorations/ broken prostheses, mild dental trauma such as uncomplicated crown fractures) - seen within seven days of presentation or calling the unit.

The standard set for this audit was that $100 \%$ of patients in the 'emergency' and 'urgent' categories, and 90\% of those in the 'routine' category should be seen within the recommended time limits. A modified proforma was devised to collect this information. Information on 43 patients/ episodes of emergency care was collected over a five-day period. Of these:

- Eight patients/episodes were categorised as Emergency Care, of which 50\% were seen within the recommended time limit of one hour. Emergency patients were called back, on average, after 7.1 minutes, and seen, on average, after two hours and 30 minutes
- Fourteen were categorised as Urgent Care, of which 100\% were seen within the recommended time limit of 24 hours. These patients were called back, on average, after 3.9 minutes, and seen, on average, after six hours and 47 minutes

- Twenty-one were categorised as Routine Care, of which 95\% were seen within the recommended time limit of seven days. These patients were called back, on average, after 11.5 hours, and seen, on average, after 36 hours.

Difficulties in offering patients care within the appropriate time limits was felt to have occurred due to a lack of available appointments with student operators, available clinic space, availability of staff to provide treatment, and dependence on patients to be accurate when reporting the nature of their condition while on the telephone. Recommendations from this audit have included developing a new proforma/ series of questions, as well as training for those taking telephone calls to ask patients seeking emergency care, so that patients are more accurately entered into their appropriate care category.

Examples of other recently completed audit projects within the community-based clinical teaching programme not detailed here include:

- Choices of restorative materials for new and replacement restorations

- Adherence to NHS/local policies on uniform wearing

- Patient satisfaction with complete dentures

- Utilisation of single-use burs

- Use of radiographs in endodontics.

\section{CONCLUDING REMARKS}

Clinical audit is now an essential and established part of dental practice. Appropriately used, it can improve the quality of care delivered, while improving patient safety. Embedding the philosophy of an appropriate approach to clinical audit within dental school teaching programmes is appropriate for preparing the next generation of dental practitioners. Through the educational experiences they create, dental school educators, along with colleagues in vocational/dental foundation training have much to offer their students in preparation for successful careers as independent dental practitioners.
1. Miller A G. Making the most of audit. Dent Update 2003; 30: 542-546.

2. A First Class Service - Quality in the New NHS. London: Department of Health, 1998.

3. Modernising NHS dentistry - clinical audit and peer review in the GDS. London: Department of Health, 2001.

4. Patel J, Fox K, Grieveson B, Youngson C C. Undergraduate training as preparation for vocational training in England: a survey of vocational dental practitioners' and their trainers' views. Br Dent J 2006; 201: 9-15.

5. Honey J, Lynch C D, Burke F M, Gilmour A S M. Ready for practice? A study of confidence levels of final year dental students Cardiff University and University College Cork. Eur J Dent Educ 2011; 15: 98-103.

6. Cabot $L B$, Radford D R. A personal view: are graduates as good as they used to be? Br Dent J 1999; 186: 318-319.

7. Lynch C D, Ash P J, Chadwick B L, Treasure E T. Community-based clinical teaching. Br Dent J 2009; 207: 141.

8. Davies B R, Leung A N, Dunne S M. Perceptions of a simulated general dental practice facility: reported experiences from past students at the Maurice Wohl General Dental Practice Centre 2001-2008. Br Dent J 2009; 207: 371-376.

9. Craddock H L. Outreach teaching - the Leeds experience: reflections after one year. Br Dent J 2008; 204: 319-324.

10. Smith M, Lennon M A, Brook A H, Robinson P G. A randomized controlled trial of outreach placement's effect on dental students' clinical confidence. J Dent Educ 2006; 70: 566-570.

11. Lynch C D, Ash P J, Chadwick B L. Student perspectives and opinions on their experience at an undergraduate outreach dental teaching centre at Cardiff: a 5-year study. Eur J Dent Educ 2010; 14: 12-16.

12. Lynch C D, Ash P J, Chadwick B L, Hannigan A. Evaluation of a UK community based clinical teaching/outreach programme by former dental students two and five years after graduation. J Dent Educ 2010; 74: 1146-1152.

13. Lynch C D, Ash P J, Chadwick B L, Hannigan A. Effect of community-based clinical teaching programs on student confidence: a view from the United Kingdom. J Dent Educ 2010; 74: 510-516.

14. General Dental Council. The first five years: the undergraduate dental curriculum, 3rd ed. (interim). London: General Dental Council, 2008.

15. Cowpe J, Plasschaert A, Harzer W, Vinkka-Puhakka $\checkmark$, Walmsley A D. Profile and competences for the graduating European dentist - update 2009. Eur J Dent Educ 2010; 14: 193-202.

16. Whaites E A. Essentials of dental radiography and radiology. Edinburgh: Churchill Livingstone, 2007.

17. The lonising Radiation (Medical Exposure) Regulations 2000. London: Department of Health. Available from: http://www.dh.gov. uk/en/Publicationsandstatistics/Publications/ PublicationsPolicyAndGuidance/DH_4007957. Accessed 15 October 2010.

18. Carrotte PV, Winstanley R B, Green J R. A study of the quality of impressions for anterior crowns received at a commercial dental laboratory. Br Dent J 1993; 174: 235-240.

19. Lynch $C D$, Allen P F. Quality of communication between dental practitioners and dental technicians for fixed prosthodontics in Ireland. J Oral Rehabil 2005; 32: 901-905

20. Jenkins S J, Lynch C D, Sloan A J, Gilmour A S M. Quality of prescription and fabrication of singleunit crowns by general dental practitioners in Wales. J Oral Rehabil 2009; 36: 150-156.

21. Kaufman $E$, Weinstein P, Milgrom P. Difficulties in achieving local anaesthesia. J Am Dent Assoc 1984; 108: 205-208

22. Keetley A, Moles D R. A clinical audit into the success rate of inferior alveolar nerve block analgesia in general dental practice. Prim Dent Care 2001; 8: $139-142$.

23. Scottish Dental Clinical Effectiveness Programme (Emergency Dental Care). 2007. Available from: http://www.sdcep.org.uk/index.aspx?o=2335 (accessed 15 October 2010). 